\title{
The Overestimation of Vancomycin-Associated Nephrotoxicity: The Effect of Rhabdomyolysis and Nephrotoxicants at a Referral Poison Center, Tehran, Iran
}

\author{
Simin Dokht Shoaei, ${ }^{1}$ Mohammad Sistanizad, ${ }^{2}$ Naser Mozafari, ${ }^{3}$ Tahereh Alinia, ${ }^{4}$ and Haleh Talaie ${ }^{5,}$ \\ ${ }^{1}$ Clinical Research and Development Center, Imam Hossein Hospital, Shahid Beheshti University of Medical Sciences, Tehran, IR Iran \\ ${ }^{2}$ Department of Clinical Pharmacy, Faculty of Pharmacy, Shahid Beheshti University of Medical Sciences, Tehran, IR Iran \\ ${ }^{3}$ Plastic Surgery Department, 15 Khordad Hospitals, Shahid Beheshti University of Medical Sciences, Tehran, IR Iran \\ ${ }^{4}$ Students' Research Office, Epidemiology Department, Health Faculty, Shahid Beheshti University of Medical Sciences, Tehran, IR Iran \\ ${ }^{5}$ Toxicological Research Center, Department of Clinical Toxicology, Loghman-Hakim Hospital, Shahid Beheshti University of Medical Sciences, Tehran, IR Iran \\ "Corresponding author: Haleh Talaie, Toxicological Research Center, Department of Clinical Toxicology, Loghman-Hakim Hospital, Shahid Beheshti University of Medical \\ Sciences, Tehran, IR Iran. Tel: +98-9122394067, Fax: +98-2155418175, E-mail: Talaie@sbmu.ac.ir
}

Received 2016 June 13; Revised 2016 September 20; Accepted 2016 September 20.

\begin{abstract}
Background: Vancomycin is a first-line therapy for infections due to Methicillin-Resistant Staphylococcus aureus (MRSA). Nephrotoxicity subsequent to vancomycin administration has been discussed in many previous researches.

Objectives: The present study aimed to determine the nephrotoxic potential of vancomycin among ventilator-associated pneumonia (VAP) poisoned patients after restricting the effect of risk factors such as rhabdomyolysis and other nephrotoxicants.

Methods: This two-year cross-sectional retrospective study was conducted among VAP patients, who received vancomycin at the toxicological intensive care unit of Loghman Hakim hospital of Iran from 2013 to 2015 . Baseline and laboratory data of eligible patients were extracted from medical records. Nephrotoxicity was defined based on the risk, injury, failure, loss, and end state (RIFLE) criteria.

Results: One hundred and fifty-four VAP patients' profiles were reviewed, of whom 110 were eligible. The median age was 33.50 (12 to 94 ) years and 73.6\% were male. The median time interval between poisoning and admission was four (0 to 48 ) hours. The most common cause of poisoning was opioids (33\%). Ten patients developed new-onset nephrotoxic event, including four in risk, four in injury and two in failure class. Median vancomycin treatment time until a nephrotoxic event was three days. There was no significant difference between those who developed nephrotoxicity compared to those who did not except median vancomycin trough level (14.5 in nephrotoxic versus 13.7 in non-nephrotoxic, $\mathrm{P}=0.007)$.

Conclusions: The result of this study indicated that nephrotoxicity rate among patients treated with vancomycin is under the influence of the poisoning by nephrotoxicants. Higher vancomycin trough level was associated with increasing nephrotoxicity rate.
\end{abstract}

Keywords: Vancomycin, Ventilator-Associated Pneumonia, Nephrotoxicity

\section{Background}

Methicillin-Resistant Staphylococcus aureus (MRSA), a leading cause of invasive infections is one of the most important causes of nosocomial pneumonia, which increases morbidity, mortality and health care resources usage. Vancomycin is a first-line therapy for infections due to MRSA, recommended by the infectious diseases society of america (IDSA) (1,2). The prevalent use of intensive vancomycin dosing schedule, which was endorsed by the latest guideline, has increased debates of its unfavorable effects (3). Nephrotoxicity subsequent to the vancomycin administration is discussed in many previous researches (4-6).

A literature review showed the range incidence of vancomycin-induced nephrotoxicity to be between 5 and 35 (7). The incidence of vancomycin-induced nephrotoxicity is highly variable depending on the ward to which the patients are admitted. Critically ill patients are usually more vulnerable to renal failure due to the severity of their illness (8-10). The good thing is that vancomycinassociated nephrotoxicity has a reversible nature, which means small likelihood of permanent damage. This is the case if the dosing is ceased and the dose is modified in the right way immediately after the occurrence of renal failure (11).

An elevated production of reactive oxygen species and oxidative stress has been reported as a mechanism for vancomycin-associated nephrotoxicity (6). Other prob- 
able risk factors of vancomycin-induced nephrotoxicity have also been suggested. The trough level in excess of $15 \mathrm{mg} / \mathrm{liter}$ was found to substantially increase the risk of a nephrotoxic event (5). Treatment duration is a factor that raises the probability of a nephrotoxic event, with most episodes occurring after seven days of therapy (5), and ranges between 4.3 and 17 days after initiation of vancomycin therapy (5). Additionally, prolonged length of hospital stay is more likely associated with nephrotoxic event (12). Some medical factors are likely to affect the Vancomycin trough concentration such as concomitant treatment with nephrotoxic agents, and admission to an intensive care unit $(5,13)$.

In humans, nephrotoxicity due to vancomycin monotherapy with typical dosage regimens is uncommon, is usually reversible, and occurs with an incidence only slightly above what is reported with other antimicrobials not considered to be nephrotoxic (14).

\section{Objectives}

The present study aimed to determine the pure nephrotoxicity potential of vancomycin amongst ventilator-associated pneumonia (VAP) and according to our rhabdomyolysis rate in poisoned patients, addressing this issue seems necessary. These observed results could have important clinical implications regarding initial trough concentrations as a prognostic indicator for nephrotoxicity and identifying patients that require careful monitoring.

\section{Methods}

\subsection{Study Design and Population}

A retrospective study was conducted for VAP patients, who received vancomycin in toxicological intensive care units (ICUs) of Loghman Hakim hospital during the last two years (2013 - 2015). In this study, all VAP patients' profiles were reviewed and only eligible patients remained in the final analysis. The medical profiles of all patients during this time span were retrieved, which summed up to 154 patients. Loghman Hakim hospital poison center (LHHPC) is the largest poison center in Iran with more than 65 years of experience in managing poisoned patients. The annual reference to this center has been estimated to be 25000 patients a year (15). The research ethics committee of Shahid Beheshti University, Tehran, Iran, approved the study protocol (94.10.20-13). Data confidentiality was considered.

Non-hemolyzed serum samples were collected from eligible individuals, and analysis was done by Roche diagnostic kits in Cobas Integra 400 plus instrument. Our lab- oratory equipment were calibrated by Preciser TDM I calibrator (includes six calibrators) and calibration intervals occurred each Lot, every 10 weeks and as required following quality control procedures.

Patient baseline and laboratory data were extracted from medical records by a trained nurse using a structured data instrument. Baseline variables included age, gender, weight, admission diagnosis, and APACHE II (acute physiology and chronic health evaluation II) score at admission, treatment received while in ICU, vancomycin doses and trough level. Information pertaining to patient comorbidities was also collected. This study only used the collected data from the patient's profile and data confidentiality was considered. The final outcome of hospitalization including recovery or death was recorded for all patients.

The definition of nephrotoxicity is on the basis of the risk, injury, failure, loss, and end state (RIFLE) criteria developed in 2004 (16). Estimated glomerular filtration rate and creatinine clearance were not used as a measure of renal function, as changes from baseline creatinine are adequately sensitive in detecting nephrotoxicity (17). Serum Creatinine ( $\mathrm{SCr}$ ) concentrations and urine output were recorded for the duration of vancomycin administration. Patients were classified accordingly to the maximum RIFLE class (risk, injury or failure), reached during their third and seventh day of vancomycin treatment commence. It is worth mentioning that hemodialysis was done twice for one patient, so we did not have any classes of loss and endstage in this study.

\subsection{Statistics}

Continuous data are reported as mean \pm standard deviation (SD) for normal distributions, and median (range) for non-normal distributions. The normality was checked statistically. Categorical data are presented as counts (\%). Initial vancomycin trough values were considered as continuous variables. Univariate comparisons were made between those with and without nephrotoxicity, using nonparametric tests of significance. Analysis was performed using the SPSS version 20.0 software (IBM Corp., Armonk, NY, USA).

\section{Results}

In this study, 154 VAP patient's profiles were reviewed, of whom 110 were eligible to remain in the final analysis.

The patients were included in the present study if they 1, had definite VAP; 2 , received vancomycin for $>72$ hour; 3 , had $\geq 1$ vancomycin trough level collected within 96 hours of vancomycin therapy; and 4 , had aSCr level of $<1.3$ 
$\mathrm{mg} / \mathrm{dL}$ at initial onset of vancomycin. Patients were omitted from final analysis if they 1, had a diagnosis of cystic fibrosis, rhabdomyolysis, diabetes mellitus, severe hypotension, bed sore, sepsis and organ failure or history of chronic hemodialysis; 2 , weight $\geq 100$ kilogram; or 3 , concomitantly received nephrotoxic agent such as aminoglycosides, colistin and amphotericin B angiotensin converting enzyme inhibitors, diuretics, amphotericin, iodinated contrast agents, acyclovir, cyclosporine and non-steroidal anti-inflammatory drugs. In this study, 154 VAP patient's profile were reviewed, of whom 110 were eligible to remain in the final analysis. The reasons for exclusion of 44 patients were rhabdomyolysis, ATN (Acute tubular necrosis) and nephrotoxic medications.

The causes of exclusion of 44 patients, according to our criteria were rhabdomyolysis, ATN and nephrotoxic medications. The median age was 33.50 (12 to 94 ) years and $73.6 \%$ ( 81 of 110 ) were male. The median time interval between poisoning and admission was four ( 0 to 48 ) hours. The most common cause of poisoning was opioids (33\%). The mean APACHE II score was $16.41 \pm 4.18$. Eighteen (16.4\%) and three $(2.7 \%)$ patients died at the ICU and general ward, respectively. The baseline characteristics of the samples are summarized in Table 1.

In spite of VAP, the other sources of infection for patients receiving vancomycin were bloodstream catheterrelated $(18.2 \%, \mathrm{n}=20)$ and genitourinary $(30.9 \%, \mathrm{n}=34)$. The median baseline SCr level was $0.9 \mathrm{mg} / \mathrm{dL}$.

Ten patients (9.1\%) developed new-onset of kidney injury following vancomycin administration, including four in risk, four in injury and two in the failure class. Median of vancomycin treatment time to kidney injury was three days.

There was no significant difference between those, who developed nephrotoxicity compared to those, who did not than vancomycin trough level.

It is important to mention that median vancomycin trough concentrations were significantly higher in nephrotoxic patients compared to non-toxic patients $(\mathrm{P}=0.007)$.

Table 2 summarizes the key differences between the nephrotoxic and non- nephrotoxic groups.

\section{Discussion}

The present study determined the prevalence of vancomycin-associated nephrotoxicity among poisoned patients with VAP attributed to MRSA (Methicillin resistant S. aureus); the patients were restricted based on the exclusion criteria to limit some confounders and biases. Nephrotoxicity rate was $9 \%$ and all were reversible. In most of our nephrotoxic patients, risk and injury were more common than failure based on the RIFLE (Risk, Injury, Failure, Loss of kidney function, and End-stage kidney disease) criteria.

Noticeably, patient's condition and kidney function affected its pharmacokinetics, volume of distribution and clearance (18). Early life cycle of vancomycin, significant adverse effects such as infusion-related toxicities, nephrotoxicity and ototoxicity were related to impurities of original formulations (19). Despite the wide variety of nephrotoxicity rate, vancomycin-induced nephrotoxicity was considered to be infrequent around 5\% and reversible. Although several evaluations noted vancomycin nephrotoxicity rates as high as 35\%, particularly among patients with vancomycin trough values of $10 \mathrm{mg} / \mathrm{L}$, the associations between vancomycin exposure and nephrotoxicity were largely attributed to baseline differences in disease severity and concomitant nephrotoxins use (19-21)

In comparison with the study of Cano et al., the incidence of nephrotoxicity (15.4\%) in intensive care unit patients, who received vancomycin for the treatment of VAP was higher than our results (22). In our previous study, we evaluated VAP poisoned patients treated with vancomycin and the incidence of renal toxicity (32\%), which was within the range of recent researches utilizing standard definition of nephrotoxicity $(11 \%-42 \%)(6,18)$. This rate was an overestimation, as we did not exclude the effects of influential factors such as rhabdomyolysis and concomitant nephrotoxic agents. Rhabdomyolysis is a common event in poisoned patients, which elevates creatinine levels. It was prevalent in a previous study on the admission day. Thus creatinine increase may be attributed to either vancomycin administration or rhabdomyolysis (18). As we excluded those patients with $\mathrm{SCr} \geq 1.3$ at initial ingestion of vancomycin, the reported prevalence in the present study did not contribute to poisoning by nephrotoxicants such as alcohol and amphetamine. Lodise et al. restricted their sample to those with baseline $\mathrm{SCr}$ level $<2$ reached to $12.6 \%$ nephrotoxicity, which is a little higher than ours (19). This may be a consequence of including those with $\mathrm{SCr}$ level < 1.3 in this study.

Although the rate of vancomycin-induced nephrotoxicity was extremely variable, the relationship between vancomycin trough and nephrotoxicity was largely uniform across studies $(5,23)$. The results of this study showed that patients, who developed nephrotoxicity were significantly more likely to have greater median vancomycin serum trough concentrations than patients, who did not develop nephrotoxicity $(\mathrm{P}=0.007)$. Also, our patients' initial vancomycin trough value ranged from $9.8 \mathrm{mg} / \mathrm{L}$ to 14.9. Parallel to our findings, Jeffres et al. reported that the observed occurrence of renal toxicity was greatest among patients with higher initial vancomycin serum trough value, but 
Table 1. Summary of Baseline Characteristics of Patients Included in the Final Analysis ${ }^{a}$

\begin{tabular}{|c|c|}
\hline Factors & $\mathbf{n}=\mathbf{1 1 0}$ \\
\hline Age, median (range) & 33.50 (12 to 94$)$ \\
\hline Lag from poisoning to admission, hours median, range & $4(0$ to 48$)$ \\
\hline Length of hospital stay & $13(5$ to 63$)$ \\
\hline Duration of vancomycin therapy, days median, range & $6(2$ to 18$)$ \\
\hline Vancomycin level, median, range & $13.70(9.80$ to 14.90$)$ \\
\hline APACHE II, mean $\pm S D$ & $16.41 \pm 4.18$ \\
\hline Gender, male & $81(73.6)$ \\
\hline Death at the ICU & $18(16.4)$ \\
\hline Death in Ward & $3(2.7)$ \\
\hline AKI & $10(9.1)$ \\
\hline Dependence on dialysis & $1(0.9)$ \\
\hline \multicolumn{2}{|l|}{ Type of poisoning, No. (\%) } \\
\hline Opioid & $33(30)$ \\
\hline Amphetamine & $6(5.5)$ \\
\hline Antidepressant and anticonvulsant & $15(13.6)$ \\
\hline Toxins & $11(10)$ \\
\hline $\mathrm{CO}$ & $2(1.8)$ \\
\hline Alcohol & $2(1.8)$ \\
\hline Multi drugs or substances & $41(37.3)$ \\
\hline
\end{tabular}

${ }^{\mathrm{a}}$ Toxins include aluminum phosphate, Organophosphate, paraquat.

Table 2. Comparison of Patients Who Developed Nephrotoxicity Versus Those Who Did Not, While Receiving Vancomycin at the Intensive Care Unit ${ }^{\mathrm{a}}$

\begin{tabular}{|c|c|c|c|}
\hline Variable & Nephrotoxic $^{b}(\mathbf{n}=10)$ & Non-Nephrotoxic $(n=100)$ & P Value \\
\hline Age, median (range) & $42.5(16$ to 84$)$ & $33(12$ to 94$)$ & 0.740 \\
\hline Lag from poisoning to admission, hours median (range) & $6(5$ to 8$)$ & $3(0$ to 48$)$ & 0.078 \\
\hline Length of hospital stay day, median (range) & $19(8$ to 32$)$ & $13(5-63)$ & 0.651 \\
\hline Duration of vancomycin therapy, days median (range) & $4.5(2$ to 12$)$ & $6(2$ to 17$)$ & 0.564 \\
\hline Vancomycin level, median (range) & $14.3(13.80$ to 14.90$)$ & 13.70 (9.8 to 14.90$)$ & 0.007 \\
\hline APACHE II, mean \pm SD) & $16.62 \pm 3.88$ & $16.54 \pm 3.88$ & 0.764 \\
\hline Gender, male, No. (\%) & $7(70)$ & $74(74)$ & 0.722 \\
\hline Outcome, No. (\%) & & & 0.183 \\
\hline Death in ICU & $4(40)$ & $14(14)$ & \\
\hline Death in Ward & 0 & $3(3.3)$ & \\
\hline
\end{tabular}

${ }^{\mathrm{a}}$ Reported as mean $\pm \mathrm{SD}$, as data was normally distributed.

${ }^{\mathrm{b}}$ Reported as median (range), as data was non-normally distributed.

initial vancomycin trough value around $10 \mathrm{mg} / \mathrm{L}$ was considered as a border of renal toxicity (24). Similarly, Lodise et al. considered vancomycin trough values as the preferable pharmacodynamic index for describing the association be- tween vancomycin exposure and toxicity (8). This is consist with another investigation, in which patients with the highest initial vancomycin trough value (during the first 96 hours of therapy) $\geq 9.9 \mathrm{mg} / \mathrm{L}$ had a significantly higher 
incidence of nephrotoxicity compared to those with values of $<9.9 \mathrm{mg} / \mathrm{L}$. Vancomycin serum trough concentration threshold from approximately $10 \mathrm{mg} / \mathrm{L}$ to $20 \mathrm{mg} / \mathrm{L}$ has been associated with the development of renal toxicity. It has been suggested that the relationship between higher trough levels and nephrotoxicity event is true in the early days of vancomycin therapy and after this point it is uncommon and was typically reversible (25).

In this study, we surveyed the nephrotoxicity of vancomycin in poisoned patients with rhabdomyolysis and VAP for the first time. One of the strengths of the present study was exclusion of patients with weight of $>100 \mathrm{Kg}$. Previous researches showed a positive association between greater weight and nephrotoxicity development $(8,19)$. Likewise, in a recent guideline, initial weight-based dosing and following adjustment based on serum trough level was recommended (26). Therefore, in the current study, vancomycin was ingested $1 \mathrm{~g}$ every 12 hours for all patients and dose adjustment was performed up to $1 \mathrm{~g}$ every 96 hon, the basis of SCr and trough level. According to another study, the rate of nephrotoxicity was significantly higher among patients, who received $>4 \mathrm{~g}$ of vancomycin daily than patients, who received less than $4 \mathrm{~g}$ (19).

Jeffres et al. mentioned that APACHE II scores as a marker of the illness severity were greater in patients developing renal toxicity, while we did not detect any significant difference between mean APACHE II scores of nephrotoxic and non-nephrotoxic patients (24). We did not observe any other significant differences between nephrotoxic and non-nephrotoxic patients based on gender, age, duration of therapy and pantoprazole concomitant administration.

We did not check the SCrafter on the seventh day of vancomycin treatment that seems to be our study limitation; however most of the patients developed nephrotoxicity so far.

\subsection{Conclusion}

The result of this study indicated that Nephrotoxicity rate among vancomycin treated patients was under the influence of poisoning by nephrotoxicants. High vancomycin trough levels had an important role in increasing nephrotoxicity rate. Further investigations are required to determine which amongst vancomycin levels and nephrotoxicity is the cause and which is the effect.

\section{Acknowledgments}

This study was supported by a grant from toxicological research center, Loghman-Hakim hospital, Shahid Beheshti University of Medical Sciences, Tehran, Iran. We gratefully acknowledge the clinical research development center and laboratory ward of the Loghman Hakim hospital, Shahid Beheshti University of Medical Sciences, Dr. Jalalizad (Nephrologist), Dr. Mohammadreza Hajiesmaeili (Anesthesiologist), Dr. Arezou Mahdavinejad (Researcher), Ms Behjat Barari (Data collector), Ms Helya-sadat Mortazavi (Researcher).

\section{Footnote}

Conflict of Interests: The authors declare that there was no conflict of interests regarding the publication of this paper.

\section{References}

1. Liu C, Bayer A, Cosgrove SE, Daum RS, Fridkin SK, Gorwitz RJ, et al. Clinical practice guidelines by the infectious diseases society of america for the treatment of methicillin-resistant Staphylococcus aureus infections in adults and children. Clin Infect Dis. 2011;52(3):18-55. doi: 10.1093/cid/ciq146. [PubMed: 21208910].

2. Burnham JP, Burnham CD, Warren DK, Kollef MH. Impact of time to appropriate therapy on mortality in patients with vancomycinintermediate staphylococcus aureus infection. Antimicrob Agents Chem. 2016;60(9):5546-53.

3. van Hal SJ, Lodise TP, Paterson DL. The clinical significance of vancomycin minimum inhibitory concentration in Staphylococcus aureus infections: a systematic review and meta-analysis. Clin Infect Dis. 2012;54(6):755-71. doi:10.1093/cid/cir935. [PubMed: 22302374].

4. Fullmer A, McCue D, Feng C. Retrospective review of vancomycininduced nephrotoxicity in patients with leukemia. J Oncol Pharm Pract. 2014;20(6):403-8. doi: 10.1177/1078155213509847. [PubMed: 24177356].

5. van Hal SJ, Paterson DL, Lodise TP. Systematic review and metaanalysis of vancomycin-induced nephrotoxicity associated with dosing schedules that maintain troughs between 15 and 20 milligrams per liter. Antimicrob Agents Chemother. 2013;57(2):734-44. doi: 10.1128/AAC.01568-12. [PubMed: 23165462].

6. Elyasi S, Khalili H, Dashti-Khavidaki S, Mohammadpour A. Vancomycin-induced nephrotoxicity: mechanism, incidence, risk factors and special populations. A literature review. Eur J Clin Pharmacol. 2012;68(9):1243-55. doi: 10.1007/s00228-012-1259-9. [PubMed: 22411630].

7. Elyasi S, Khalili H, Hatamkhani S, Dashti-Khavidaki S. Prevention of vancomycin induced nephrotoxicity: a review of preclinical data. Eur J Clin Pharmacol. 2013;69(4):747-54. doi: 10.1007/s00228-012-1406-3. [PubMed: 22996076].

8. Lodise TP, Lomaestro B, Graves J, Drusano GL. Larger vancomycin doses (at least four grams per day) are associated with an increased incidence of nephrotoxicity. Antimicrob Agents Chemother. 2008;52(4):1330-6. doi: 10.1128/AAC.01602-07. [PubMed:18227177].

9. Eswarappa M, Gireesh MS, Ravi V, Kumar D, Dev G. Spectrum of acute kidney injury in critically ill patients: A single center study from South India. Indian J Nephrol. 2014;24(5):280-5. doi: 10.4103/09714065.132991. [PubMed: 25249716].

10. Piccinni P, Cruz DN, Gramaticopolo S, Garzotto F, Dal Santo M, Aneloni $\mathrm{G}$, et al. Prospective multicenter study on epidemiology of acute kidney injury in the ICU: a critical care nephrology Italian collaborative effort (NEFROINT). Minerva Anestesiol. 2011;77(11):1072-83. [PubMed: 21597441]. 
11. Zimmermann AE, Katona BG, Plaisance KI. Association of vancomycin serum concentrations with outcomes in patients with gram-positive bacteremia. Pharmacotherapy. 1995;15(1):85-91. [PubMed: 7739950].

12. Minejima E, Choi J, Beringer P, Lou M, Tse E, Wong-Beringer A. Applying new diagnostic criteria for acute kidney injury to facilitate early identification of nephrotoxicity in vancomycin-treated patients. Antimicrob Agents Chemother. 2011;55(7):3278-83. doi: 10.1128/AAC.0017311. [PubMed: 21576448].

13. Rybak MJ, Lomaestro BM, Rotschafer JC, Moellering RC, Craig WA Billeter M, et al. Vancomycin therapeutic guidelines: a summary of consensus recommendations from the infectious diseases Society of America, the American Society of Health-System Pharmacists, and the Society of Infectious Diseases Pharmacists. Clin Infect Dis. 2009;49(3):325-7. doi:10.1086/600877. [PubMed: 19569969].

14. Rybak M, Lomaestro B, Rotschafer JC, Moellering RJ, Craig W, Billeter $\mathrm{M}$, et al. Therapeutic monitoring of vancomycin in adult patients: a consensus review of the American Society of Health-System Pharmacists, the Infectious Diseases Society of America, and the Society of Infectious Diseases Pharmacists. Am J Health Syst Pharm. 2009;66(1):8298. doi: 10.2146/ajhp080434. [PubMed: 19106348].

15. Hassanian-Moghaddam H, Zamani N, Rahimi M, Shadnia S, Pajoumand A, Sarjami S. Acute adult and adolescent poisoning in Tehran, Iran; the epidemiologic trend between 2006 and 2011. Arch Iran Med. 2014;17(8):534-8. doi: 014178/AIM.003. [PubMed: 25065275].

16. Bellomo R, Ronco C, Kellum JA, Mehta RL, Palevsky P, Acute Dialysis Quality Initiative W. Acute renal failure - definition, outcome measures, animal models, fluid therapy and information technology needs: the Second International Consensus Conference of the Acute Dialysis Quality Initiative (ADQI) Group. Crit Care. 2004;8(4):R204-12. doi:10.1186/cc2872. [PubMed: 15312219].

17. Pickering JW, Endre ZH. Back-calculating baseline creatinine with MDRD misclassifies acute kidney injury in the intensive care unit. Clin J Am Soc Nephrol. 2010;5(7):1165-73. doi: 10.2215/CJN.08531109. [PubMed: 20498242].

18. Kouchek M, Asghari R, Mahdavinejad A, Salimi A, Barari B, Seyedrazi $\mathrm{P}$, et al. Comparative study between teicoplanin and vancomycin in methicillin-resistant staphylococcus aureus (mrsa) infectious of tox- icological intensive care unit (ticu) patients-tehran iran. Life Sci J 2014;11(3s):83-90.

19. Lodise TP, Patel N, Lomaestro BM, Rodvold KA, Drusano GL. Relationship between initial vancomycin concentration-time profile and nephrotoxicity among hospitalized patients. Clin Infect Dis. 2009;49(4):507-14. doi:10.1086/600884. [PubMed: 19586413].

20. Cimino MA, Rotstein C, Slaughter RL, Emrich LJ. Relationship of serum antibiotic concentrations to nephrotoxicity in cancer patients receiving concurrent aminoglycoside and vancomycin therapy. Am J Med. 1987;83(6):1091-7.

21. Elting LS, Rubenstein EB, Kurtin D, Rolston K, Fangtang J, Martin C, et al. Mississippi mud in the 1990s. Cancer. 1998;83(12):2597-607.

22. Cano EL, Haque NZ, Welch VL, Cely CM, Peyrani P, Scerpella EG, et al. Incidence of nephrotoxicity and association with vancomycin use in intensive care unit patients with pneumonia: retrospective analysis of the IMPACT-HAP Database. Clin Ther. 2012;34(1):149-57. doi: 10.1016/j.clinthera.2011.12.013. [PubMed: 22284995].

23. Matson KL, Shaffer CL, Beck GL, Simonsen KA. Assessment of initial serum vancomycin trough concentrations and their association with initial empirical weight-based vancomycin dosing and development of nephrotoxicity in children: a multicenter retrospective study. Pharmacotherapy. 2015;35(3):337-43. doi: 10.1002/phar.1552. [PubMed: 25756622].

24. Jeffres MN, Isakow W, Doherty JA, Micek ST, Kollef MH. A retrospective analysis of possible renal toxicity associated with vancomycin in patients with health care-associated methicillin-resistant Staphylococcus aureus pneumonia. Clin Ther. 2007;29(6):1107-15. doi: 10.1016/j.clinthera.2007.06.014. [PubMed:17692725].

25. Prabaker KK, Tran TP, Pratummas T, Goetz MB, Graber CJ. Elevated vancomycin trough is not associated with nephrotoxicity among inpatient veterans. J Hosp Med. 2012;7(2):91-7. doi: 10.1002/jhm.946. [PubMed: 22086511].

26. Ackerman BH, Guilday RE, Reigart CL, Patton ML, Haith LR. Evaluation of the relationship between elevated vancomycin trough concentrations and increased efficacy and/or toxicity. J Burn Care Res. 2013;34(1):1-9. doi: 10.1097/BCR.ob013e3182676ee8. [PubMed: 23292591]. 